\title{
Optical and NIR spectroscopy of Mrk 1210: constraints and physical conditions of the active nucleus $\star, \star \star$
}

\author{
X. Mazzalay ${ }^{1}$ and A. Rodríguez-Ardila ${ }^{2, \star \star \star}$ \\ ${ }^{1}$ IATE, Observatorio Astronómico Córdoba, Laprida 854, X5000BGR, Córdoba, Argentina \\ e-mail: ximena@oac . uncor .edu \\ 2 Laboratório Nacional de Astrofísica, Rua dos Estados Unidos 154, Itajubá, MG, Brazil
}

Received 13 September 2005 / Accepted 22 August 2006

\section{ABSTRACT}

\begin{abstract}
Aims. Mrk 1210 is an outstanding Seyfert 2 galaxy because it displays signatures of recent circumnuclear star formation and a high level of X-ray activity, in addition to the classical spectral characteristics typical of an AGN. Here we investigate the extinction affecting the nuclear and extended emitting gas, the kinematics of the narrow-line region, and the physical properties and conditions of that gas.

Methods. Near-infrared and optical spectra of the nuclear and extended emission region of Mrk 1210 are presented, covering the interval 0.4-2.4 $\mu \mathrm{m}$. Emission and absorption lines were used to infer, respectively, the geometrical extension of the ionized gas and the contribution of the underlying stellar population to the observed integrated continuum. The emission line profiles were employed to study the kinematics in the NLR. The reddening and physical condition of the gas were investigated by means of flux ratios among permitted and forbidden lines.

Results. The NIR nuclear spectrum is dominated by HI and He I recombination lines, as well as [S II], [S III], and [Fe II] forbidden lines. Coronal lines of [S VIII], [S IX], [Si VI], [Si X], and [Ca VIII], in addition to molecular $\mathrm{H}_{2}$ lines, were also detected. The ${ }^{12} \mathrm{CO}(6-3) 1.618 \mu \mathrm{m}$ overtone bandhead helped to estimate the contribution of the stellar population to the continuum. It was found that $83 \pm 8 \%$ of the $H$-band continuum has a stellar origin. It improves previous estimates, which claimed that at least $50 \%$ of the observed continuum was attributed to the AGN. Analysis of the emission line profiles, both allowed and forbidden, shows a narrower $\left(F W H M \sim 500 \mathrm{~km} \mathrm{~s}^{-1}\right)$ line on top of a broad $\left(F W H M>1000 \mathrm{~km} \mathrm{~s}^{-1}\right)$ blue-shifted component. This seems to be associated to a nuclear outflow. This hypothesis is supported by $6 \mathrm{~cm}$ VLBI observations, which show a radio ejecta extending up to $\sim 30 \mathrm{pc}$ from the nucleus. This result does not require the presence of the hidden BLR claimed to be present in previous NIR observations of this object. Internal extinction, calculated by means of several indicators including [Fe II] flux ratios not previously used before in AGNs, reveals a dusty AGN, while the extended regions are barely affected by dust, if at all. The density and temperature are calculated for the NLR using optical and NIR lines as diagnostic ratios. The results show electronic temperatures from $10000 \mathrm{~K}$ up to $40000 \mathrm{~K}$ and densities between $10^{3}-10^{5} \mathrm{~cm}^{-3}$. The higher temperatures show that shocks, most probably related to the radio outflow, must contribute to the line emission.
\end{abstract}

Key words. galaxies: active - galaxies: individual: Mrk 1210 - galaxies: Seyfert - infrared: galaxies

\section{Introduction}

The source Mrk 1210, also known as the Phoenix galaxy, is a nearby Sa Seyfert 2 galaxy at $z=0.01350$. It has been extensively studied in the optical region, mainly because of both the Wolf-Rayet features found within the central $200 \mathrm{pc}$ (Storchi-Bergmann et al. 1998), indicating the presence of a circumnuclear starburst, and the detection of broad $\mathrm{H} \alpha$ and $\mathrm{H} \beta$ components in polarized light (Tran et al. 1992; Tran 1995). The widths of these last components reach $2380 \mathrm{~km} \mathrm{~s}^{-1}$ at FWHM. Near-infrared (NIR) spectra reported by Veilleux et al. (1997) show that the $\mathrm{Pa} \beta$ profile is characterized by a strong

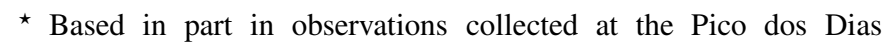
Observatory/LNA, Brazil.

$\star \star$ Figures 1-3 are only available in electronic form at http: //www . aanda. org

$\star \star \star$ Visiting Astronomer at the Infrared Telescope Facility, which is operated by the University of Hawaii under Cooperative Agreement no. NCC 5-538 with the National Aeronautics and Space Administration, Office of Space Science, Planetary Astronomy Program. narrow component on top of a broad base with FWHM $1600 \mathrm{~km} \mathrm{~s}^{-1}$, suggesting the presence of a hidden broad-line region (BLR). However, a comparison of the $\mathrm{Pa} \beta$ profile with that of [Fe II] $1.256 \mu \mathrm{m}$ indicates that at least part of the broad emission is also present in the forbidden [Fe II] line. The moderate critical densities of the NIR [Fe II] lines, $n_{\mathrm{c}} \sim 10^{4-5} \mathrm{~cm}^{-3}$ (Nisini et al. 2002), and the broad emission observed in [Fe II] $1.256 \mu \mathrm{m}$ suggest that the broad permitted line is not produced in a genuine high-density BLR. The possible presence of a broad component to $\mathrm{Pa} \beta$ and $\mathrm{Br} \gamma$ and the absence of broad $\mathrm{H} \alpha$ in direct light would imply an $E(B-V)_{\mathrm{b}}>1.6$ and 1.1 , respectively, meaning that this object is strongly reddened.

Additional evidence of the dusty nature of Mrk 1210 is abundant. It is a member of the sample of galaxies called $60 \mu \mathrm{m}$ peakers (60PKs, Heisler \& De Roberties 1999) because of its "warm" far-infrared color and the spectral energy distribution peaking near $60 \mu \mathrm{m}$. Over the past few years, evidence has been accumulating indicating that these properties can be attributed to a dust-obscured active galactic nucleus (e.g., Keel et al. 1994; Hes et al. 1995; Heisler \& Vader 1995). Whether or not this 
obscured material is the putative dusty torus, as postulated by the unified model (e.g., Antonucci 1993), is highly debatable although evidence from spectropolarimetry points to this scenario. In fact, Heisler et al. (1997) showed that the Seyfert 2 galaxies for which polarized broad lines have been detected have warmer far-infrared colors $\left(f_{60} / f_{25}<4\right)$ compared to Seyfert 2 galaxies for which polarized broad lines have not been detected. The warm IRAS Seyferts are believed to be viewed at an angle that is more face-on than that of cool IRAS Seyferts. Moreover, in the near-infrared, the degree of polarization of Mrk 1210 rises toward longer wavelengths, reaching $\sim 5 \%$ at $2.15 \mu \mathrm{m}$ (Watanabe et al. 2003). Observational evidence suggests that this polarization is due to dichroic absorption by aligned grains. Watanabe et al. (2003) also suggest the existence of an additional unpolarized component, most probably associated to electron scattering.

The picture of a highly reddened nucleus derived from polarimetry is also strongly supported by optical multicolor imaging. Indeed, Heisler \& Vader (1994) showed that Mrk 1210 displays a smooth global light distribution but morphologically resembles a typical elliptical galaxy. Masked frames display arcs or shells surrounding the main body of the galaxy. More recently, Martini et al. (2003) classified Mrk 1210 as a tightly wound nuclear dust spiral with the individual dust lanes traced for over a full rotation about the nucleus.

In X-rays, Mrk 1210 is peculiar. It is one of the very few cases of an AGN caught in a dramatic transition between a Compton-thick, reflection dominated state, and a Compton-thin state (Guainazzi et al. 2002). This result was possible after comparing XMM Newton observations with ASCA observations made six years earlier. The transition is attributed to either a change in the properties of the absorbers or a "switching-off" of the nucleus during the ASCA observations. In the former scenario, it may indicate a clumpy or patchy structure of the torus to which the absorbers are associated, while the latter may provide clues to the duty-cycle of the AGNs phenomenon.

All the above means that Mrk 1210 is a key source where the interplay between an active nucleus and the presence of strong star formation can be studied in detail. Therefore, it is a very important object for investigating the nuclear structure of Seyfert 2 galaxies. In order to achieve this goal, it is necessary to characterize these two components (i.e., nuclear and stellar) as completely as possible. The presence of broad-emission line components, a high degree of polarization, power law near-infrared colors, warm far-infrared spectrum, high radio-brightness temperature in the milli-arcsecond central structure, and finally, hard $\mathrm{X}$-ray emission, all suggest that a significant contribution comes from the active nucleus. This paper is the first attempt to study the properties in Mrk 1210 that can be attributed to the AGN, although not leaving aside the stellar contribution. Because of the strong evidence of dust obscuration, the NIR region is most appropriate because it attenuates only a fraction $(\sim 1 / 7)$ of the light compared to that in the optical, allowing an unbiased estimate of the obscured AGN to be obtained.

Here, we study Mrk 1210 by means of JHK spectroscopy covering the interval $0.8-2.4 \mu \mathrm{m}$, complemented by optical spectroscopy aimed at investigating (i) the extinction affecting the nuclear and extended emitting gas; (ii) the kinematics of the narrow line region (NLR); and (iii) the physical properties and conditions of that gas. To meet the first goal, a variety of extinction diagnostic ratios based on permitted and forbidden lines, some of them never used before in an AGN, are used. This will allow us to make a detailed map of the dust distribution on that object. For the second goal, a detailed comparison of the emission line profiles, both in the optical and NIR, are carried out. It will also allow us to state whether or not a hidden broad-line region is in fact present in the spectra of this object. For the third goal, density and temperature-sensitive line ratios are employed to construct a detailed picture of the ionization structure of emitting gas. In Sect. 2 we describe the observations and data reduction process. Section 3 describes the most important emission features found in the NIR spectra, some of them reported for the first time in this work. Section 4 discusses the kinematics of the NLR gas, while Sect. 5 evaluates and discusses the dust distribution in the nuclear and extended emission regions of Mrk 1210. Section 6 explores the physical condition throughout the narrow-line region, and concluding remarks are found in Sect. 7. A Hubble constant of $75 \mathrm{~km} \mathrm{~s}^{-1} \mathrm{Mpc}^{-1}$ is used in this work.

\section{Observations and data reduction}

\subsection{Infrared data}

Near-infrared spectra in the interval $0.8-2.4 \mu \mathrm{m}$ were obtained at the NASA 3 m Infrared Telescope Facility (IRTF) on April 21, 2002 with the SpeX facility spectrometer (Rayner et al. 2003). The detector consisted of a $1024 \times 1024 \mathrm{InSb}$ array, with a spatial scale of $0.15^{\prime \prime} /$ pixel. A $0.8^{\prime \prime} \times 15^{\prime \prime}$ slit was used during the observations, providing a spectral resolution $R \sim 900$. The slit was oriented at the paralactic angle $\left(60^{\circ}\right)$ in order to minimize losses by atmospheric refraction. The standard nodding technique ABBA was employed during the observations. This procedure allows a good cancellation of sky features and the removal of the bias and dark levels. After the target, observed with an effective airmass of 1.22, spectra of the AOV star HD 10961 were taken at a similar airmass (1.15) to cancel out the telluric bands and flux-calibrate the source spectra. Seeing was $0.7^{\prime \prime}$ during the integrations. The spectral extraction and calibration procedures were performed using Spextool, the inhouse software developed and provided by the SpeX team for the IRTF community (Cushing et al. 2004). When reducing the data, each $A B$ source pair was subtracted from each other to remove sky features, and the resulting frames were combined to provide a final 2-D spectrum. Five 1-D spectra were extracted along the spatial direction by summing up the signal in a window size of $1^{\prime \prime}$ each. The spectrum associated to the nuclear region (hereafter nuclear spectrum, corresponding to the inner $250 \mathrm{pc}$ ) was centered in the maximum of the light profile distribution. Two additional spectra, adjacent to the nuclear one (250 pc SW and NE of the center, AP1 and AP3, respectively) were extracted on either side of the light-peak distribution. The last two spectra correspond to regions located at $500 \mathrm{pc}$ SW and NE (AP2 and AP4, respectively) of the center. Note that at the distance at which Mrk 1210 is located, $1^{\prime \prime}=250 \mathrm{pc}$.

Telluric absorption correction and flux calibration were applied to the individual 1-D spectra by means of the IDL based routine xtellcor (Vacca et al. 2003). Xtellcor makes use of the spectrum of the A0V observed after the source (see above) and a high resolution model of Vega, to construct a telluric correction spectrum that is free of telluric absorption features. The flux calibration is done by knowing the magnitude of the telluric standard and using the reddening curve of Rieke \& Lebofsky (1985) in order to estimate the flux as a function of wavelength for the given star. This response function is then applied to the target.

Final reduced spectra were corrected for redshift, as determined from the average $z$ measured from the positions of [S III] $0.953 \mu \mathrm{m}$, He I $1.083 \mu \mathrm{m}$, [Fe II] $1.256 \mu \mathrm{m}$, [CI] $0.985 \mu \mathrm{m}, \mathrm{Br} \gamma$ and $\mathrm{Pa} \gamma$. A galactic extinction correction 
of $E(B-V)=0.030$ was applied to the data (Schlegel et al. 1998). The reduced spectra in the interval $0.8-2.4 \mu \mathrm{m}$ are plotted, in rest wavelengths, in Figs. 1 and 2 for the nuclear and extended regions, respectively.

\subsection{Optical data}

In addition to the NIR data, optical spectra covering the wavelength interval 3900-6900 ̊ were taken using two different configurations and detectors. The observations were done with the Cassegrain spectrograph attached to the $1.6 \mathrm{~m}$ telescope at the Observatório Pico dos Dias, Brazil. In both cases, a slit width of $2^{\prime \prime}$, oriented east-west was employed. The spectrum containing the $\mathrm{H} \alpha$ line (5100-6900 $\AA$, red spectrum hereafter) was obtained on November 28, 2002 (UT) using a $1024 \times 1024$ CCD and a $600 \mathrm{l} / \mathrm{mm}$ grating that provided a spectral resolution of $4.5 \AA$. The observation covering $\mathrm{H} \beta$ (3900-5300 $\AA$, blue spectrum hereafter) was taken on May 7, 2003 (UT) with a $2048 \times 2048 \mathrm{CCD}$ and a $900 \mathrm{l} / \mathrm{mm}$ grating. The spectral resolution obtained with the latter configuration was $2.4 \AA$. Data were reduced following IRAF standard techniques, that is, bias subtraction and flat field division. Wavelength calibration were done using HeNeAr lamps. For flux calibration, a spectrophotometric standard was observed after the targets. Due to bad seeing $\left(\sim 2^{\prime \prime}\right)$, the procedure for extracting the blue and red spectra was to integrate all the signal along the slit. After reduction and flux calibration, the continuum in the overlap region (5100-5300 $\AA$ ) disagreed. In order to bring both spectra to the same continuum level, a scale factor of 0.74 was applied to the blue spectrum. This number corresponds to the ratio of the integrated flux of [NI] $5200 \AA$ for the red and blue spectra. Note that [NI] was chosen because it is the only line common to both spectra. A final reduced optical spectrum is plotted in Fig. 3.

Throughout this article, wavelengths corresponding to the optical data, from the blue up to $8000 \AA$ will be given in Angstroms. The NIR wavelengths longwards of to $8001 \AA$ will be reported in microns.

\subsection{Emission line fluxes}

In order to measure the flux of the emission lines in the different spectra, we assumed that the line profiles can be represented either by a single or by a sum of Gaussian profiles. The continuum underneath each line was fit by a low-order polynomium, usually a straight line. The LINER routine (Pogge \& Owen 1993) was employed in this process. Typically, most emission lines were well fitted by a single or a sum of two Gaussian components. Fluxes for the detected lines or upper limits found in the nuclear and extended regions in the near-infrared are listed in Tables 1 and 2. Table 3 reports the measurements on the optical spectra. In all cases, errors correspond to $3 \sigma$ above the continuum level and reflect the uncertainty in the placement of the continuum and in the $\mathrm{S} / \mathrm{N}$ around the line of interest. When a line identification was uncertain, the Atomic Line List Database ${ }^{1}$ was consulted to select a list of candidates. A final identification was possible according to the presence or absence of additional lines of the candidate ion. Additional support for line identification was obtained by comparing the position of the emission lines with those observed in planetary nebulae, for instance.

Due to the differences in spatial resolution between the optical and NIR spectra, we summed up the fluxes of each line

\footnotetext{
1 Available at http://www.pa.uky.edu/ peter/atomic/
}

measured in the five apertures of the NIR data for all the cases where the analysis involved the simultaneous use of optical and NIR lines. This procedure was applied for consistency and guarantees that the size of both optical and NIR apertures are representative of the same emitting region.

\section{The near-infrared spectrum of Mrk 1210}

The nuclear spectrum of Mrk 1210 displays a plethora of emission lines, from those emitted from molecular $\mathrm{H}_{2}$ and low and medium ionization species such as [CI], [S II], and [S III], up to very high ionization lines of [S IX] and [Si X]. This implies a very wide range of physical conditions of the emitting gas, even within the central $250 \mathrm{pc}$, to allow the simultaneous coexistence of molecules, somehow protected from the intense radiation of the central engine, and highly ionized gas, undoubtedly associated to the AGN phenomenon. In addition, absorption lines of $\mathrm{Ca}$ II and $\mathrm{CO}$ are also observed, indicating the presence of a circumnuclear stellar population.

The off-nuclear spectra of Mrk 1210 are also peculiar. At $250 \mathrm{pc} \mathrm{NE}$ and $\mathrm{SW}$ of the nucleus, emission from $\mathrm{HI}$ and He I dominates. Farther out, at 500 pc from the center, both the NE and SW spectra show emission from He I, [Fe II], and $[\mathrm{S} \mathrm{III}]$. Weak $\mathrm{Pa} \beta$ is detected in the 500 pc NE, suggesting that the extended emission is dominated by matter-bounded clouds. The detection of extended [S III] is compatible with the data presented by Fraquelli et al. (2003) who show extended [O III] $5006 \AA$ up to $1 \mathrm{kpc}$ from the nucleus.

\subsection{The forbidden NIR spectrum}

The brightest forbidden lines observed in the nuclear spectrum of Mrk 1210 are [S III] $0.953 \mu \mathrm{m}$ and [S III] $0.906 \mu \mathrm{m}$. In addition, Fig. 2 shows that the former line is not limited to the nuclear region. It extends up to $500 \mathrm{pc} \mathrm{NE}$ and $250 \mathrm{pc}$ SW from the center. In the AP2 spectrum, a feature at the expected position of [S III] $0.953 \mu \mathrm{m}$ is detected but the $\mathrm{S} / \mathrm{N}$ is low. For this reason, we only report an upper limit for its flux.

An inspection of Fig. 1 shows that Mrk 1210 displays a very rich coronal NIR spectrum. Here, we report the first detection of [S VIII] $0.991 \mu \mathrm{m}$, [S IX] $1.252 \mu \mathrm{m}$, [Si X] $1.430 \mu \mathrm{m}$, and [Ca VIII] $2.320 \mu \mathrm{m}$ on this object. The strongest coronal line observed is [Si VI] which is, to our knowledge, the only one previously reported for this galaxy in the $0.8-2.4 \mu \mathrm{m}$ range (Rodríguez-Ardila et al. 2004). At 250 pc from the nucleus, high-ionization lines such as [S VI] $1.964 \mu \mathrm{m}$, and [SiX] $1.430 \mu \mathrm{m}$ are still visible, mostly in the SW direction. This, however, cannot be taken as a definitive probe of extended emission in the coronal lines. It is possible that they represent scattered nuclear photons or even that they are emitted in the inner few tens of parsecs of the extended region.

In addition to the features mentioned above, Mrk 1210 is characterized by a conspicuous [Fe II] spectrum, not previously reported in the literature for other AGN. Besides [Fe II] $1.256 \mu \mathrm{m}$ and $1.643 \mu \mathrm{m}$, which is common in most Seyferts (Rodríguez-Ardila et al. 2004) and whose flux ratio is an excellent reddening indicator for the NLR, up to eight more lines are detected in the $J$ and $H$-bands. The presence of these lines is a prime opportunity to use the forbidden iron lines as a diagnostic tool for the region where they are formed. In Sect. 5 we will explore the diagnostic capabilities of the [Fe II] as a probe of the physical conditions of the NLR. As already mentioned, 
Table 1. Line identifications and fluxes for the infrared spectra.

\begin{tabular}{|c|c|c|c|c|c|c|c|c|c|}
\hline \multirow[b]{2}{*}{ Ion } & \multirow[b]{2}{*}{$\lambda(\mu \mathrm{m})$} & \multicolumn{3}{|c|}{ Flux* } & \multirow[b]{2}{*}{ Ion } & \multirow[b]{2}{*}{$\lambda(\mu \mathrm{m})$} & \multicolumn{3}{|c|}{ Flux* } \\
\hline & & AP1 & NUC & AP3 & & & AP1 & NUC & AP3 \\
\hline [Fe II $]$ & 0.861 & - & $4.7 \pm 0.1$ & - & {$[\mathrm{Fe} \mathrm{II}]$} & 1.256 & $2.7 \pm 0.2$ & $11.2 \pm 0.1$ & $2.2 \pm 0.1$ \\
\hline [S III] & 0.906 & $11.0 \pm 0.4$ & $50.0 \pm 1.5$ & $7.6 \pm 0.5$ & [Fe II] & 1.270 & - & $1.0 \pm 0.2$ & - \\
\hline [Fe II] & 0.923 & - & $3.8 \pm 0.3$ & - & $\mathrm{Pa} \beta$ & 1.281 & $4.8 \pm 0.3$ & $23.6 \pm 0.3$ & $3.6 \pm 0.4$ \\
\hline$[\mathrm{Fe} \mathrm{II}]$ & 0.946 & - & $1.6 \pm 0.5$ & - & {$[\mathrm{Fe} \mathrm{II}]$} & 1.294 & $0.2 \pm 0.1$ & $2.2 \pm 0.1$ & $0.5 \pm 0.2$ \\
\hline [S III] & 0.953 & $25.6 \pm 0.5$ & $120.1 \pm 0.8$ & $22.7 \pm 0.5$ & {$[\mathrm{Fe}$ II $]$} & 1.297 & $0.3 \pm 0.1$ & $0.7 \pm 0.1$ & $0.2 \pm 0.1$ \\
\hline$[\mathrm{C} \mathrm{I}]$ & 0.982 & $0.5 \pm 0.3$ & $1.3 \pm 0.2$ & $0.3 \pm 0.2$ & {$[\mathrm{Si} \mathrm{X}]$} & 1.430 & $0.7 \pm 0.2$ & $2.8 \pm 0.4$ & - \\
\hline$[\mathrm{C} \mathrm{I}]$ & 0.985 & $1.0 \pm 0.3$ & $3.3 \pm 0.2$ & $0.8 \pm 0.2$ & [Fe II] & 1.533 & $0.6 \pm 0.3$ & $2.8 \pm 0.1$ & - \\
\hline [S VIII] & 0.991 & - & $2.9 \pm 0.2$ & - & [Fe II] & 1.643 & $1.9 \pm 0.2$ & $12.5 \pm 0.5$ & $1.7 \pm 0.2$ \\
\hline $\mathrm{Pa} \delta$ & 1.004 & $1.6 \pm 0.4$ & $5.3 \pm 0.5$ & $1.0 \pm 0.3$ & [Fe II] & 1.676 & $0.5 \pm 0.1$ & $2.5 \pm 0.3$ & $0.5 \pm 0.1$ \\
\hline He II & 1.012 & $2.4 \pm 0.4$ & $7.1 \pm 0.3$ & $1.0 \pm 0.3$ & $\operatorname{Pa} \alpha$ & 1.875 & & & \\
\hline [S II] & 1.028 & $2.8 \pm 0.3$ & $11.3 \pm 0.9$ & $1.8 \pm 0.2$ & $\operatorname{Br} \delta$ & 1.944 & $0.5 \pm 0.3$ & $3.8 \pm 1.1$ & - \\
\hline [S II] & 1.032 & $2.8 \pm 0.3$ & $12.2 \pm 0.9$ & $2.7 \pm 0.2$ & $\mathrm{H}_{2}$ & 1.957 & $0.7 \pm 0.3$ & $2.5 \pm 0.3$ & $0.5 \pm 0.1$ \\
\hline [S II] & 1.033 & $2.3 \pm 0.3$ & $12.2 \pm 0.9$ & $0.9 \pm 0.2$ & [Si VI] & 1.964 & $1.9 \pm 0.4$ & $13.3 \pm 0.9$ & $1.6 \pm 0.2$ \\
\hline [S II $]$ & 1.037 & $1.0 \pm 0.3$ & $5.6 \pm 0.9$ & $0.8 \pm 0.2$ & $\mathrm{H}_{2}$ & 2.033 & - & $0.5 \pm 0.1$ & - \\
\hline [N I] & 1.040 & $2.3 \pm 0.3$ & $8.7 \pm 1.0$ & $1.1 \pm 0.2$ & $\mathrm{He} \mathrm{I}$ & 2.058 & - & $1.3 \pm 0.3$ & - \\
\hline He I & 1.083 & $37.8 \pm 0.5$ & $179.3 \pm 0.5$ & $30.0 \pm 0.4$ & $\mathrm{H}_{2}$ & 2.121 & $0.2 \pm 0.1$ & $1.6 \pm 0.8$ & $0.10 \pm 0.03$ \\
\hline $\mathrm{Pa} \gamma$ & 1.093 & $2.5 \pm 0.4$ & $10.5 \pm 0.5$ & $1.9 \pm 0.5$ & $\mathrm{Br} \gamma$ & 2.165 & $0.8 \pm 0.1$ & $4.6 \pm 0.2$ & $0.6 \pm 0.3$ \\
\hline [P II] & 1.146 & - & $2.3 \pm 0.3$ & - & $\mathrm{H}_{2}$ & 2.223 & $0.3 \pm 0.1$ & $0.9 \pm 0.1$ & $0.15 \pm 0.07$ \\
\hline $\mathrm{H}_{2}$ & 1.185 & - & $0.25 \pm 0.14$ & - & $\mathrm{H}_{2}$ & 2.247 & - & $0.2 \pm 0.1$ & - \\
\hline [P II] & 1.188 & - & $3.7 \pm 0.3$ & - & [Ca VIII] & 2.320 & - & $1.6 \pm 0.1$ & - \\
\hline [S IX] & 1.252 & $1.0 \pm 0.2$ & $4.2 \pm 0.5$ & $0.5 \pm 0.2$ & & & & & \\
\hline
\end{tabular}

* In units of $10^{-15} \mathrm{erg} \mathrm{cm}^{-2} \mathrm{~s}^{-1}$.

Table 2. Line identifications and fluxes in the NIR for the AP2 and AP4.

\begin{tabular}{lccc}
\hline \hline & & \multicolumn{2}{c}{ Flux $^{*}$} \\
\cline { 3 - 4 } Ion & $\lambda(\mu \mathrm{m})$ & AP2 & AP4 \\
\hline$[$ S III $]$ & 0.953 & $<0.53$ & $0.60 \pm 0.3$ \\
He I & 1.083 & $1.08 \pm 0.4$ & $0.97 \pm 0.15$ \\
{$[\mathrm{Fe}$ II] } & 1.256 & $<0.63$ & $<0.2$ \\
\hline
\end{tabular}

* In units of $10^{-15} \mathrm{erg} \mathrm{cm}^{-2} \mathrm{~s}^{-1}$.

Table 3. Line identifications and fluxes for the optical spectra.

\begin{tabular}{lcclcc}
\hline \hline Ion & $\lambda(\AA)$ & Flux $^{*}$ & Ion & $\lambda(\AA)$ & Flux $^{*}$ \\
\hline$[\mathrm{Ne}$ III $]$ & 3967 & $22.8 \pm 9.0$ & {$[\mathrm{~N} \mathrm{II}]$} & 5754 & $6.3 \pm 1.0$ \\
{$[\mathrm{~S} \mathrm{II}]$} & 4068 & $36.4 \pm 6.8$ & $\mathrm{He}$ I & 5875 & $8.0 \pm 1.0$ \\
$\mathrm{H} \delta$ & 4101 & $9.3 \pm 6.2$ & {$[\mathrm{Fe}$ VII $]$} & 6086 & $9.5 \pm 1.2$ \\
$\mathrm{H} \gamma$ & 4340 & $22.2 \pm 3.3$ & {$[\mathrm{O} \mathrm{I}]$} & 6300 & $45.8 \pm 1.5$ \\
{$[\mathrm{O}$ III $]$} & 4363 & $22.2 \pm 4.0$ & {$[\mathrm{~S} \mathrm{III}]$} & 6312 & $13.2 \pm 1.0$ \\
$\mathrm{He}$ II & 4685 & $10.3 \pm 2.6$ & {$[\mathrm{O} \mathrm{I}]$} & 6363 & $33.0 \pm 1.5$ \\
$\mathrm{H} \beta$ & 4861 & $72.6 \pm 3.5$ & {$[\mathrm{~N} \mathrm{II}]$} & 6548 & $19.0 \pm 1.3$ \\
{$[\mathrm{O}$ III $]$} & 4958 & $221.8 \pm 2.2$ & $\mathrm{H} \alpha$ & 6562 & $419.3 \pm 1.8$ \\
{$[\mathrm{O}$ III $]$} & 5006 & $636.4 \pm 2.2$ & {$[\mathrm{~N} \mathrm{II}]$} & 6583 & $57.0 \pm 1.3$ \\
{$[\mathrm{~N} \mathrm{I}]$} & 5197 & $4.0 \pm 0.6$ & {$[\mathrm{~S} \mathrm{II}]$} & 6716 & $38.4 \pm 1.1$ \\
{$[\mathrm{O}$ I] } & 5577 & $<1.8$ & {$[\mathrm{~S} \mathrm{II}]$} & 6730 & $42.4 \pm 1.1$ \\
{$[\mathrm{Fe}$ VII $]$} & 5720 & $8.3 \pm 2.0$ & & & \\
\hline
\end{tabular}

* In units of $10^{-15} \mathrm{erg} \mathrm{cm}^{-2} \mathrm{~s}^{-1}$.

we found that [Fe II] $1.256 \mu \mathrm{m}$ extends up to $500 \mathrm{pc}$ NE and SW from the center (see Fig. 2).

Other interesting features on the NIR forbidden spectrum are [S II] $1.028 \mu \mathrm{m}, 1.032 \mu \mathrm{m}, 1.033 \mu \mathrm{m}$, and $1.037 \mu \mathrm{m}^{2}$. These lines, along with the optical doublet [S II] 4068, $4076 \AA$ arise from the same upper term. It means that the flux ratio between the NIR and optical sulfur lines depends primarily on their

${ }^{2}$ We will use the term [S II] $1.03 \mu \mathrm{m}$, hereafter, to indicate the sum of the fluxes of these four sulfur lines. transition probabilities. Therefore, it can be used as an additional reddening indicator.

Finally, low ionization lines of [N I $],[\mathrm{C} \mathrm{I}]$, and [P II] are also detected in the nuclear spectrum. With exception of the later, these lines are also observed in AP1 and AP3 (see Fig. 2). In the nucleus, the flux ratio $[\mathrm{PII}] /[\mathrm{CI}]$ is 1.3 (see Table 1 ). The detection of [P II] lines of considerable strength is surprising. This is because in the sun, phosphorus is a factor $\sim 1000$ times less abundant than carbon. Therefore, if the $\mathrm{P} / \mathrm{C}$ abundance is even closer to solar, the [P II] lines should not be present unless carbon and other strong abundant elements are much more optically thick than they appear. An alternative to this apparent contradiction is to assume that phosphorus is over-abundant by a factor 10-30 compared to its solar abundance, relative to hydrogen. A similar problem is found in high-redshift quasars, where broad absorption lines of P V $\lambda \lambda 1118,1128 \AA$ are detected (Hamman 1998; Hamman et al. 2002). The NIR [P II] lines may probably help to set some constraints to the abundance of phosphorus in AGN. This subject, however, is outside of the scope of this paper.

\subsection{Molecular and absorption lines}

Several molecular hydrogen lines are present in the nuclear and extended NIR spectra of Mrk 1210, mostly in the $K$-band. We detected $\mathrm{H}_{2}$ emission up to a distance of $250 \mathrm{pc}$, both $\mathrm{NE}$ and SW from the center. An inspection to the spectra of Figs. 1 and 2 shows that the strongest $\mathrm{H}_{2}$ lines observed in this galaxy are $(1,0) S(1)$ at $2.121 \mu \mathrm{m}$ and $(1,0) S(3)$ at $1.957 \mu \mathrm{m}$. Also detected in the $K$-band, but less intense, are $(1,0) S(2) 2.033 \mu \mathrm{m}$, $(1,0) S(1) 2.223 \mu \mathrm{m}$, and $(2,1) S(1) 2.247 \mu \mathrm{m}$. These lines were studied by Rodríguez-Ardila et al. (2004) in a sample of AGN, including Mrk 1210, aimed at determining the dominant $\mathrm{H}_{2}$ excitation mechanisms. They found that, for Mrk 1210, the $\mathrm{H}_{2}$ is predominantly excited by stellar processes (UV heating), with some contribution of fluorescence $(\sim 30 \%)$ and 
little influence from X-ray heating (the dominant mechanism for most AGN). This result is supported by the presence of both a circumnuclear starburst (Schulz \& Henkel 2003) and strong $\mathrm{H}_{2} \mathrm{O}$ megamaser emission coming from a small region around the nucleus (Braatz \& Wilson 1994) that can be associated to star-forming regions. The vibrational and rotational temperatures derived by Rodríguez-Ardila et al. (2004) for the nuclear $\mathrm{H}_{2}$ gas were $3100 \pm 700 \mathrm{~K}$ and $900 \pm 300 \mathrm{~K}$, respectively.

The flux of the $\mathrm{H}_{2}(1,0) S(1) 2.121 \mu \mathrm{m}$, listed in Table 1 for the different apertures and corrected for reddening (see Sect. 5), can be used to derive the mass of hot molecular gas present in the inner few hundred parsecs of Mrk 1210. To this purpose, we used the expression:

$m_{\mathrm{H}_{2}} \simeq 5.0875 \times 10^{13} D^{2} I_{1-0 S(1)}$

taken from Reunanen et al. (2002) and assuming $T=2000 \mathrm{~K}$, a transition probability $A_{S(1)}=3.47 \times 10^{-7} \mathrm{~s}^{-1}$ (Turner et al. 1977), and the population fraction in the $v=1, J=3$ level $f_{v=1, J=3}=0.0122$ (Scoville et al. 1982). $I_{1-0 S(1)}$ is the flux of $\mathrm{H}_{2} 2.121 \mu \mathrm{m}$ corrected for intrinsic $E(B-V)$.

The values of the $\mathrm{H}_{2}$ mass found are $260 \pm 40 M_{\odot}$ for the nucleus, $30 \pm 13 M_{\odot}$ for AP1 and $14 \pm 4 M_{\odot}$ AP3, totalizing nearly $300 M_{\odot}$ of hot molecular gas for the inner $500 \mathrm{pc}$ of Mrk 1210. It is in very good agreement with the nuclear $\mathrm{H}_{2}$ masses reported by Reunanen et al. (2003) for a sample of Seyfert 1 and 2 galaxies. The small mass of hot $\mathrm{H}_{2}$ contrasts with the cold molecular mass of $7.94 \times 10^{8} M_{\odot}$ found by Raluy et al. (1998) from $\mathrm{CO}(1 \rightarrow 0)$ observations and using the standard conversion factor found in Galactic giant molecular clouds, $M_{\mathrm{H}_{2}}=5.8 L_{\mathrm{CO}}$. The aperture of the $\mathrm{CO}$ data covers the inner $5.7 \mathrm{kpc}$. Interestingly, Raluy et al. (1998) found high starformation efficiency in Mrk 1210 (derived from the $L_{\mathrm{IR}} / M_{\mathrm{H}_{2}}$ ratio), comparable to that of normal galaxies like the Milky Way or M 33. In fact, from a list of 12 AGNs, Mrk 1210 is the one that shows the second highest star-formation efficiency. They, however, called attention to the fact that a significant fraction of the IR luminosity (up to 50\%) could come from the AGN itself, resulting in overestimation of the star formation efficiency by up to a factor of 2 .

A rough estimate of the contribution of the stellar population flux to the $H$-band can be made by measuring the depth of the ${ }^{12} \mathrm{CO}(6-3)$ overtone bandhead at $1.618 \mu \mathrm{m}$ (see Fig. 1). We found that the observed line depth is $11 \pm 1 \%$ of the continuum. This can be compared to the $\sim 20 \%$ that is typically expected for a population of GKM supergiants that dominate the $H$-band light for stellar populations older that $10^{7} \mathrm{yr}$ (Schinnerer et al. 1998). It means that $55 \pm 5 \%$ of the total flux in the $H$-band continuum is due to GMK supergiants. Although these stars dominate the stellar continuum emission in the $H$-band, Schinnerer et al. (1998) claims that about one third of the total stellar flux is due to stars of other spectral types. Therefore, $83 \pm 8 \%$ of the $H$-band continuum in a $0.8^{\prime \prime} \times 1^{\prime \prime}$ aperture has a stellar origin. This result agrees very well with the $71 \pm 6 \%$ contribution of a stellar population older that $10^{7} \mathrm{yr}$ determined by Cid Fernandes et al. (2001) for this object, based on optical spectroscopy. It means that the $H$-band continuum is essentially dominated by the stellar population, with only $\sim 20 \%$ due to the AGN. This new value refines the claim made by Raluy et al. (1998), who estimated that at least $50 \%$ of the IR could come from the active nucleus. The above calculations suggest that NIR spectroscopy is a potential tool for unveiling hidden starburst components, either in highly obscured objects or in those in which the optical continuum emission is dominated by the AGN component (as in Seyfert 1 galaxies).
Table 4. $F W H M^{*}$ and shifts in the peak centroid of the broad component measured in the lines displaying narrow and broad components.

\begin{tabular}{lcccc}
\hline \hline & & \multicolumn{2}{c}{$F W H M\left[\mathrm{~km} \mathrm{~s}^{-1}\right]$} & \\
\cline { 3 - 4 } Ion & $\lambda(\mu \mathrm{m})$ & $\mathrm{BC}^{1}$ & $\mathrm{NC}^{2}$ & $\Delta \mathrm{V}$ \\
\hline $\mathrm{H} \beta$ & 0.486 & 1410 & 330 & 234 \\
{$[\mathrm{O}$ III] } & 0.495 & 1130 & 260 & 76 \\
{$[\mathrm{O} \mathrm{III}]$} & 0.500 & 1120 & 260 & 78 \\
{$[\mathrm{~S} \mathrm{III}]$} & 0.906 & 1260 & 460 & 114 \\
{$[\mathrm{~S} \mathrm{III}]$} & 0.953 & 1270 & 460 & 102 \\
$\mathrm{He}$ & 1.083 & 1510 & 500 & 113 \\
$\mathrm{~Pa} \beta$ & 1.281 & 1360 & 390 & 225 \\
\hline
\end{tabular}

${ }^{*}$ Corrected for instrumental resolution. ${ }^{1}$ Broad Component; ${ }^{2}$ narrow Component.

Even after correcting for the increased contribution of the stellar population to the continuum, Mrk 1210 continues to display one of the highest (if not the highest) star formation rates among the group of 12 AGN studied by Raluy et al. (1998).

The strong contribution of stellar light to the Mrk 1210 continuum in the inner $0.8^{\prime \prime 2}$ is also compatible with the analysis of the $\mathrm{H}_{2}$ lines presented in the beginning of this section. It further supports the hypothesis that in this AGN the stellar population contributes significantly to the excitation of the molecular lines.

\section{Kinematics of the narrow line region}

The large amount of spectral information at medium resolution, covering the optical and NIR regions, allows us to study the form and width of the emission lines in Mrk 1210. The purpose is to map the velocity field of the NLR, examine the presence of genuine BLR components, and estimate the most probable location of the emitting gas.

The results of the Gaussian decomposition carried out to measure the line fluxes (see Sect. 2.3) showed that most lines were well-fitted by a single narrow Gaussian component of $F H W M \sim 500 \mathrm{~km} \mathrm{~s}^{-1}$. However, bright lines such as [S III], [O III], $\mathrm{Pa} \beta$, and He I needed an additional, broader blue-shifted component (see Table 4) of $F W H M>1000 \mathrm{~km} \mathrm{~s}^{-1}$. It is known that only broad permitted lines (with $F W H M \sim 10^{3-4} \mathrm{~km} \mathrm{~s}^{-1}$ ) would be present at typical BLR electron densities $\left(n_{\mathrm{e}} \sim\right.$ $10^{9-11} \mathrm{~cm}^{-3}$ ). In this galaxy not only do the permitted lines have broad components but also the forbidden ones. Veilleux et al. (1997) reported that the $\mathrm{Pa} \beta$ and $\mathrm{Br} \gamma$ lines profiles of Mrk 1210 were characterized by a strong narrow component on top of a broad base. They compared the H I lines with [Fe II] $1.256 \mu \mathrm{m}$ and $\mathrm{H}_{2} 2.121 \mu \mathrm{m}$. Because of the smaller FWHM displayed by the last two lines, they suggested that some of the emission was produced in a genuine high-density BLR. With our data we are able to extend this comparison to a larger number of permitted and forbidden lines to see if the hidden broad-line region proposed by Veilleux et al. (1997) is in fact observable in the NIR spectrum of Mrk 1210.

Figure 4 shows a comparison of optical and NIR emission line profiles in velocity space. The upper left panel (a) shows [O III] $5006 \AA, \mathrm{H} \beta$ and $\mathrm{Br} \gamma$, while the upper right panel (b) plots [S III] $0.953 \mu \mathrm{m}$, [O III] $5006 \AA$, and $\mathrm{Br} \gamma$. It can be seen that both permitted and forbidden lines in the NIR region are quite similar in form and width, ruling out the hypothesis of a broad component associated to the BLR. Moreover, Fig. 4 shows that the optical lines are systematically narrower at FWHM than the NIR ones. In contrast, the full width at zero intensity $(F W Z I)$ are 

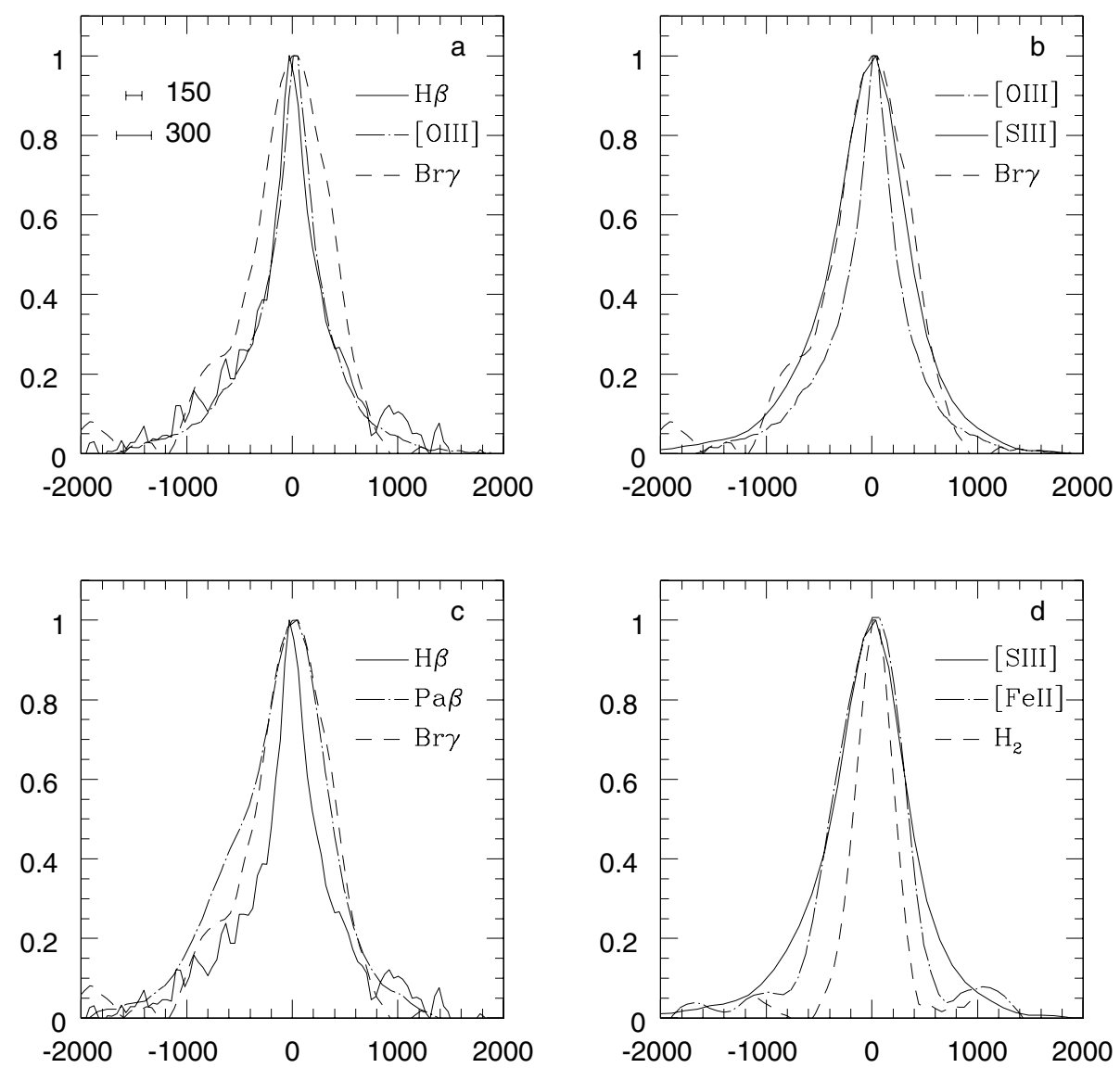

Fig. 4. Profiles of nuclear lines in Mrk 1210 in velocity space. The lines are normalized to the same peak flux. The small bars in panel a) represent the instrumental profile of the blue spectrum $\left(F W H M=150 \mathrm{~km} \mathrm{~s}^{-1}\right)$ and that of the NIR region $\left(F W H M=330 \mathrm{~km} \mathrm{~s}^{-1}\right)$.

rather similar in both regions. In panel (c) a comparison of $\mathrm{H} \beta$, $\mathrm{Pa} \beta$, and $\mathrm{Br} \gamma$ is shown. Note, again, that the $F H W M$ of the optical and NIR lines are different but the $F W Z I$ is equivalent.

The difference in $F W H M$ among the narrow components may be attributed to either selective extinction or to a difference in the instrumental resolution. In the first scenario, optical photons emitted by low-velocity clouds are more absorbed than the NIR ones, allowing us to more likely see the optical emission originated in high velocity clouds, which apparently are less subjected to extinction. In the second scenario, the difference in $F W H M$ between NIR and optical lines is due to differences in the instrumental profiles. In fact, the instrumental $F W H M$ of the NIR data is a factor of about 2.2 larger than that of the blue spectrum (instrumental $F W H M=150 \mathrm{~km} \mathrm{~s}^{-1}$ ). Although at first sight the latter argument would be sufficient to explain the differences in width, the values of FHWM listed in Table 4 point out that very likely selective extinction dominates. This is because in Table 4, the FWHM of the lines have been corrected for instrumental resolution. Clearly, all the lines are spectroscopically resolved, and most important, the narrow components of [O III] and $\mathrm{H} \beta$ are narrower than those of [S III] and $\mathrm{Pa} \beta$, for instance.

Note that the $\mathrm{Pa} \beta$ profile shows enhanced emission blueward of the line center. This feature is visible not only in Mrk 1210, but also in other Seyfert galaxies (e.g. NGC 2110, Storchi-Bergman et al. 1999; NGC 4151, Thompson 1995). We attribute this asymmetry primarily to the presence of [Fe II] $1.278 \mu \mathrm{m}$, which contaminates the blue wing of $\mathrm{Pa} \beta$. In spite of this feature, the $\mathrm{Pa} \beta$ and $\mathrm{Br} \gamma$ line profiles are rather similar, and the difference at $F W H M$ with the optical lines is clear. In panel (d) we compare [Fe II] $1.643 \mu \mathrm{m}, \mathrm{H}_{2} 2.121 \mu \mathrm{m}$ and [S III] $0.953 \mu \mathrm{m}$. Note the slight blue asymmetry in [S III], present neither in [Fe II] nor in $\mathrm{H}_{2}$. It can also be seen that from peak intensity to $F W H M$, [Fe II] and [S III] are indistinguishable. From FWHM to FWZI, the two profiles diverge, with [S III] becoming broader. In contrast, $\mathrm{H}_{2}$ is significantly narrower, almost half the $F W H M$ value of the former two. Since extinction cannot be invoked to explain these differences because all three lines are located in the NIR region, the most plausible explanation is that the bulk of [S III], [Fe II], and $\mathrm{H}_{2}$ originates in the different volumes of gas, which are subjected to different dispersion velocity conditions.

The results of the above comparison show that the same broad component present in the permitted lines is also detected in some of the forbidden lines. This indicates that the gas responsible for the broad lines is most likely associated to the NLR itself. Additional support for this picture is found from the work of Lutz et al. (2002). They compared the emission line profiles of [Si IX] $3.934 \mu \mathrm{m}$ and $\mathrm{Br} \alpha$ in Mrk 1210 and decided against the BLR interpretation because the two profiles were indistinguishable within the $\mathrm{S} / \mathrm{N}$ limitations.

We propose that the broad component seen in [S III], [O III], and the permitted lines is associated to outflows, instead of to the hidden BLR suggested by Veilleux. Evidence of this scenario comes from Middelberg et al. (2004), who carried out radio observations of Mrk 1210 at $18 \mathrm{~cm}$ and $6 \mathrm{~cm}$ with EVN, MERLIN, and VLBA. The EVN and VLBA $18 \mathrm{~cm}$ images reveal a bright compact object and a weaker component toward the southeast at a distance of $8.6 \mathrm{pc}$. They marginally detected a third component at a distance of $30.6 \mathrm{pc}$ from the bright compact source. They suggest that the last component could 
Table 5. Flux ratios and $E(B-V)$.

\begin{tabular}{|c|c|c|c|c|c|c|c|}
\hline \multirow[b]{2}{*}{ Ratio } & \multirow[b]{2}{*}{$\begin{array}{l}\text { Intrinsic } \\
\text { value }\end{array}$} & \multicolumn{2}{|c|}{ AP1 } & \multicolumn{2}{|c|}{$\overline{\mathrm{NUC}}$} & \multicolumn{2}{|c|}{ AP3 } \\
\hline & & $\begin{array}{l}\text { Measured } \\
\text { value }\end{array}$ & $E(B-V)$ & $\begin{array}{l}\text { Measured } \\
\text { value }\end{array}$ & $E(B-V)$ & $\begin{array}{l}\text { Measured } \\
\text { value }\end{array}$ & $E(B-V)$ \\
\hline [Fe II] $1.256 / 1.643 \mu \mathrm{m}$ & $1.36^{1}$ & $1.42 \pm 0.18$ & $<0.35$ & $0.89 \pm 0.04$ & $1.49 \pm 0.14$ & $1.29 \pm 0.16$ & $<0.62$ \\
\hline$[\mathrm{Fe}$ II $] 1.676 / 1.294 \mu \mathrm{m}$ & $0.77^{1}$ & $2.50 \pm 1.34$ & $4.5 \pm 2.0$ & $1.14 \pm 0.14$ & $1.49 \pm 0.49$ & $1.00 \pm 0.44$ & $<2.72$ \\
\hline$[\mathrm{Fe}$ II $] 1.533 / 1.297 \mu \mathrm{m}$ & $1.06^{1}$ & $3.0 \pm 12.0$ & $5.71 \pm 3.88$ & $1.27 \pm 0.07$ & $1.00 \pm 0.32$ & - & - \\
\hline $\mathrm{Pa} \beta / \mathrm{Pa} \gamma$ & $1.79^{2}$ & $1.92 \pm 0.33$ & $<1.08$ & $2.24 \pm 0.14$ & $1.01 \pm 0.22$ & $1.89 \pm 0.54$ & $<1.52$ \\
\hline $\mathrm{Br} \gamma / \mathrm{Pa} \delta$ & $0.50^{2}$ & $0.50 \pm 0.14$ & $<0.34$ & $0.84 \pm 0.10$ & $0.68 \pm 0.13$ & $0.60 \pm 0.35$ & $<0.94$ \\
\hline $\mathrm{Br} \gamma / \mathrm{Pa} \gamma$ & $0.31^{2}$ & $0.32 \pm 0.06$ & $<0.35$ & $0.44 \pm 0.03$ & $0.52 \pm 0.10$ & $0.31 \pm 0.18$ & $<0.88$ \\
\hline $\mathrm{Pa} \beta / \mathrm{Pa} \delta$ & $2.90^{2}$ & $3.00 \pm 0.77$ & $<0.80$ & $4.45 \pm 0.25$ & $1.15 \pm 0.25$ & $3.60 \pm 1.15$ & $<1.45$ \\
\hline $\mathrm{Pa} \gamma / \mathrm{Pa} \delta$ & $1.62^{2}$ & $1.56 \pm 0.46$ & $<1.77$ & $1.98 \pm 0.21$ & $1.37 \pm 0.72$ & $1.89 \pm 0.76$ & $<3.79$ \\
\hline $\mathrm{Br} \gamma / \mathrm{Pa} \beta$ & $0.17^{2}$ & $0.17 \pm 0.02$ & $<0.27$ & $0.19 \pm 0.01$ & $0.31 \pm 0.10$ & $0.17 \pm 0.08$ & $<1.12$ \\
\hline $\mathrm{H} \alpha / \mathrm{H} \beta$ & $3.10^{2}$ & & & $5.77 \pm 0.27$ & $0.62 \pm 0.05$ & & \\
\hline [S II] $1.03 \mu \mathrm{m} / 4068 \AA$ & $0.67^{3}$ & & & $1.55 \pm 0.39$ & $0.28 \pm 0.08$ & & \\
\hline
\end{tabular}

${ }^{1}$ Calculated from Nussbaumer \& Storey (1988) transition probabilities; ${ }^{2}$ Osterbrock (1989); ${ }^{3}$ calculated from Keenan (1991) atomic data.

probably be the continuation of the radio ejecta. Moreover, their $6 \mathrm{~cm}$ VLBA data resolves the brightest source into an arc of four components, as well as of the south east feature. Note that most of the galaxy's $6 \mathrm{~cm}$ emission comes from scales between $3 \mathrm{pc}$ and $16 \mathrm{pc}$. This region is unresolved from our data. Middelberg et al. (2004) suggest three scenarios for the radio structure in Mrk 1210. A free-free emitting disk, synchrotron emission from a torus or extended accretion disk, and a system composed of a core and an outflow. Based on physical arguments, the last hypothesis is most favored. The resolved arc of four components could be bright knots due to a shock in an extended, low brightness temperature radio outflow, with similar conditions to those in NGC 5506, NGC 1068 and Mrk 231.

An additional support for this picture is that the peak of the broad component detected in [S III], [O III], H I, and He I is blueshifted relative to the systemic velocity of the galaxy (see Table 4). The shift in the forbidden lines is similar to that of He I and almost half of HI. This broad component is probably associated to the series of four knots reported by Middelberg et al. (2004), which is not spatially resolved in our nuclear spectrum. This also would explain how the line profiles of some forbidden and permitted lines in Fig. 4 are different at FWHM but similar at FWZI. The highest dispersion velocities are associated to the outflow component, with little or no extinction toward the line of sight. At $F W H M$, the bulk of the line profile is dominated by the emission from the classical NLR clouds. The NIR lines are broader because we are looking deep into the clouds, where the dispersion velocity is higher.

\section{The extinction affecting the NLR of Mrk 1210}

The numerous $\mathrm{HI}$ and forbidden lines in the spectra of Mrk 1210, spanning a large interval in wavelength, allowed us to evaluate the intrinsic extinction affecting the NLR within the inner few hundred parsec by means of several indicators. In those cases where the determination of reddening only involved NIR lines, we were able to trace it at different distances from the nucleus, obtaining additional information about the dust distribution.

The extinction was determined from the comparison of the predicted and observed emission line ratios, assuming the standard extinction law of Cardelli et al. (1989) for $R_{V}=3.1$; note, however, that at these wavelengths, the extinction law is nearly independent of the assumed $R_{V}$. Listed in Table 5 are the line ratios used for the reddening determinations (first column), the intrinsic ratio values (second column), and the measured line ratios and estimated reddening for three infrared spectra (AP1, NUC and AP3). For AP2 and AP4, no sufficient information is available. The last two rows in Table 5 list the ratios involving optical lines. These last values correspond to an emitting region size equivalent to the one covered by the sum of the five infrared extractions $(500 \mathrm{pc})$. In all cases, the errors correspond to $3 \sigma$ and were determined by assuming that the only quantities that introduce uncertainties are the measured line fluxes.

For the hydrogen lines, we used the intrinsic ratios for case B given by Osterbrock (1989). Our results point out to extinction variations up to a factor of nearly 5 within the NLR. Recall, however, that the $E(B-V)$ derived using $\mathrm{Pa} \beta$ may be misleading. This is because of the contamination of the $\operatorname{Pa} \beta$ line with [Fe II] $1.278 \mu \mathrm{m}$, as mentioned in the previous section. This feature increases the $\mathrm{Pa} \beta$ flux, leading to an overestimation of the reddening if it is determined from the $\mathrm{Pa} \beta / \mathrm{Pa} \gamma$ ratio or to an underestimation, if it is derived from $\mathrm{Br} \gamma / \mathrm{Pa} \beta$.

A previous determination of the reddening in Mrk 1210 was carried out by Veilleux et al. (1997). They calculated a value of $E(B-V)=0.48$ using the Balmer decrement reported by Terlevich et al. (1991). Although this value is lower than the one determined here from the optical H I, the discrepancy can probably be explained in terms of seeing, aperture, and position-angle effects. Also, our result is slightly above the one found in a recent statistical work presented by Ho et al. (2003), which showed that Seyfert galaxies tend to have an internal mean reddening of about 0.43 (see also Koski 1978).

The detection of the [S II] infrared lines near $1.03 \mu \mathrm{m}$ and the optical lines at $4068,4076 \AA$, all arising from the same upper ${ }^{2} \mathrm{P}$ term, allows us to estimate the interstellar extinction affecting the $\mathrm{S}^{+}$region. This method was first pointed out by Miller (1968) and tested by Wampler $(1968,1971)$ in several Seyfert galaxies, and more recently by Greve et al. (1994) in the Orion nebula. Due to the large separation in wavelength between the NIR and optical [S II], the reddening derived from this ratio should be very accurate. Using the atomic data from Keenan (1991), we obtained an [S II] near-infrared to optical theoretical ratio of 0.67 . The observed ratio and the reddening coefficient are given in Table 5. The $E(B-V)$ of $0.28 \pm 0.08$ found for the $\mathrm{S}^{+}$region is the smallest one derived for the nuclear gas of Mrk 1210, indicating that these lines, as expected, are formed in the outer portions of the NLR. 
In addition to the extinction determined from the $\mathrm{HI}$ and [S II] lines, the rich [Fe II] spectrum detected in Mrk 1210 offers a unique opportunity to study the extinction affecting the region where it is emitted. This is because each of the three pairs of lines involved in the [Fe II] ratios listed in Table 5 share the same upper level. It means that the intensity ratio depends only on energy differences between the lines and their Einstein $A$-coefficients, making these ratios useful probes of reddening. The scarce detection of such a rich [Fe II] spectrum in other AGN has prevented its use as diagnostics of the internal extinction. For this reason, extinction estimates with the NIR [Fe II] lines are unusual and, to our knowledge, this is the first time that they have been applied to a Seyfert nucleus (except for the ratio [Fe II] $1.256 \mu \mathrm{m} / 1.643 \mu \mathrm{m}$ ). Intrinsic line ratios were taken from Bautista \& Pradhan (1998), calculated from Nussbaumer \& Storey (1988) transition probabilities.

Our results, listed in Table 5, clearly show that high values of extinction affects the [Fe II] emission region. That all three [Fe II] reddening indicators agree within the errors points out that all [Fe II] is emitted in the same dusty environment. Although the uncertainties in some ratios are large, mostly because the lines involved are intrinsically weak, the high extinction derived for the [Fe II] emitting region leads us to propose that the bulk of these lines is formed deep in the NLR, separate from the zone where other low-ionization species are emitted (i.e. [S II] and $[\mathrm{OI}])$. In contrast to the nucleus, the reddening found for AP1 and AP3 shows that the extended emission region are barely affected by dust, if at all (see Table 5).

The picture that emerges from the above analysis points out that the dust distribution in the inner 250 pc of Mrk 1210 is rather inhomogeneous. Much of the optical emission we see should be emitted in the outer portions of the NLR, while the NIR one comes from the deep NLR. This is supported by the analysis of the permitted and forbidden lines made in Sect. 4. At $F H W M$, the optical narrow components are narrower than the NIR ones. Moreover, that the broad components display a similar width in velocity space, from the visible through NIR, suggests that they are being emitted in a region not affected by dust. Whether the high extinction measured from the [Fe II] and the $\mathrm{H}$ I lines is associated to the circumnuclear starburst, to the host galaxy, or even to the torus cannot be easily distinguished from our data. However, we should recall that Martini et al. (2003) used HST images to classify Mrk 1210 as a tightly-wound nuclear dust spiral with the individual dust lanes traced over a full rotation about the nucleus. This view supports the idea that the dust is mainly concentrated in a planar geometry, probably associated to the host galaxy. In this scenario, the broad component arises in the outflow, which is inclined, from the dust plane relative to our line of sight. Further support for this scenario comes from the dramatic transition between a Comptonthick, reflection-dominated state and a Compton-thin state in Mrk 1210 reported by Guainazzi et al. (2002) from X-ray observations. They claimed that this transition can be explained if the Compton-thick and Compton-thin absorbers are different. The former is associated to the torus while the latter, located on larger scales, may be associated with the host galaxy rather than with the nuclear environment.

\section{Electron density and temperature: physical properties of the nuclear environment}

Electronic densities and temperatures for the nuclear gas of Mrk 1210 can be determined by means of several diagnostic line ratios. But before this can be done, line fluxes need to be corrected in accordance with the value of reddening obtained from lines of the same ion or similar ions. Thus, lowionization lines such as $[\mathrm{OI}],[\mathrm{NI}],[\mathrm{N}$ II] and [S II] were corrected by $E(B-V)=0.28$. This value was determined from the [S II] lines. The exception of this low-ionization group is [Fe II], whose lines were corrected by $E(B-V)=1.5$, as measured from the different indicators employed. The remaining lines were corrected for $E(B-V)=0.5$. It results from averaging out our determination of internal extinction by means of optical H I lines, the one reported by Veilleux et al. (1997) for Mrk 1210 and the average internal extinction reported by Ho et al. (2003).

In the low-density regime, the [S II] $\lambda 6716 / \lambda 6730$ flux ratio is a well-known diagnostics of the electronic density that is relatively insensitive to temperature. This diagnostic can be applied to regions where the electronic density is lower than the critical density of the $[\mathrm{S} \mathrm{II}]$ lines $\left(n_{\mathrm{c}} \sim 10^{4} \mathrm{~cm}^{-3}\right)$. Above this density the lines become collisionally de-excited. The observed [S II] ratio of 0.9 indicates an electron density $n_{\mathrm{e}} \sim 1000 \mathrm{~cm}^{-3}$ for temperatures in the interval $10000 \mathrm{~K}$ to $50000 \mathrm{~K}$. On the other hand, the infrared [S II] $1.03 \mu \mathrm{m}$ lines are observed to be quite strong relative to the $6716,6730 \AA$ doublet in the nuclei of Mrk 1210 , indicating that a second, higher density $\left(n_{\mathrm{e}} \geq 10^{4} \mathrm{~cm}^{-3}\right) \mathrm{S}^{+}$zone can also exist. The [S II] $1.03 \mu \mathrm{m}(\lambda 6716+\lambda 6730)$ line ratio is very sensitive to variations in density, in particular for values of $n_{\mathrm{e}} \geq 10^{4} \mathrm{~cm}^{-3}$ where the optical [S II] diagnostic no longer varies with $n_{\mathrm{e}}$. The observed [S II] $1.03 \mu \mathrm{m}(\lambda 6716+\lambda 6730)$ line ratio is 0.59 , which indicates an electron density $\sim 13000 \mathrm{~cm}^{-3}$ for an assumed temperature $T_{\mathrm{e}}=10000 \mathrm{~K}$ (Keenan 1991). The differences between the densities derived from the two [S II] line flux ratios illustrate that the [S II] lines observed in the spectrum of the central region of Mrk 1210 arise from different zones with different densities: a denser region $\left(\sim 13000 \mathrm{~cm}^{-3}\right)$ that produces the bulk of the [S II] $\lambda 4068, \lambda 4076$ and $1.03 \mu \mathrm{m}$ emission and a lower density region $\left(\sim 1000 \mathrm{~cm}^{-3}\right)$ that contributes with most of the $\lambda 6716$ and $\lambda 6730$ flux.

The density of the $\mathrm{Fe}^{+}$emitting region can be derived from the observed [Fe II] $1.533 \mu \mathrm{m} / 1.643 \mu \mathrm{m}$ and [Fe II] $0.861 \mu \mathrm{m} / 1.256 \mu \mathrm{m}$ line ratios (Pradhan \& Zhang 1993). The former ratio yields a density $n_{\mathrm{e}} \sim 10^{4.2} \mathrm{~cm}^{-3}$ for $T_{\mathrm{e}}$ in the interval 3000-12000 $\mathrm{K}$, while from the latter ratio we derive a higher density, $n_{\mathrm{e}} \sim 10^{5.2} \mathrm{~cm}^{-3}$, assuming $T_{\mathrm{e}}=10000 \mathrm{~K}$. These results, together with the ones from the previous sections, strongly suggest that the [Fe II] lines are indeed emitted in a separate region, characterized with higher densities and higher extinction.

The gas temperature can be estimated from measurements of pairs of emission line ratios emitted by a single ion from two levels with considerably different excitation energies. Two good examples are the [O III] $(\lambda 4958+\lambda 5006) / \lambda 4363$ and $[\mathrm{N}$ II $](\lambda 6548+\lambda 6583) / \lambda 5754$ line ratios. Temperature determinations from these ratios were calculated, using the TEMDEN task included in the STSDAS Version 3.3 package of IRAF. The atomic data employed by the routine are at least as recent as those given in the compilation by Pradhan \& Peng (1995). The results obtained for a wide range of densities are plotted in Fig. 5, which shows very high temperatures in the $\mathrm{O}^{+2}$ and $\mathrm{N}^{+}$zones, ranging from $18000 \mathrm{~K}$ up to $50000 \mathrm{~K}$. Although the ionization potential required to form the $\mathrm{O}^{+2}$ and $\mathrm{N}^{+}$ions (35.1 and $14.5 \mathrm{eV}$, respectively) are different, the critical densities for the upper levels of the lines involved in the [O III] and [N II] line ratios are $\geq 10^{4.9} \mathrm{~cm}^{-3}$. Thus it is reasonable to suppose that these lines arise from the same region with $n_{\mathrm{e}} \leq n_{\mathrm{c}}$. Therefore, we set a common temperature of $T_{\mathrm{e}}=22000 \mathrm{~K}$, for an assumed 
Table 6. Density and temperatures found from different diagnostic line ratios.

\begin{tabular}{lcc}
\hline \hline Density sensitive line ratio & Assumed temperature $[\mathrm{K}]$ & Density $\left[\mathrm{cm}^{-3}\right]$ \\
\hline$[\mathrm{S} \mathrm{II}] 6716 / 6730 \AA$ & $10000-50000$ & $\sim 10^{3.0}$ \\
{$[\mathrm{~S} \mathrm{II}] 1.03 \mu \mathrm{m} /(6716+6730) \AA$} & 10000 & $\sim 10^{4.1}$ \\
{$[\mathrm{Fe} \mathrm{II}] 1.533 / 1.643 \mu \mathrm{m}$} & $3000-12000$ & $\sim 10^{4.2}$ \\
{$[\mathrm{Fe}$ II $] 0.861 / 1.256 \mu \mathrm{m}$} & 10000 & $\sim 10^{5.2}$ \\
\hline Temperature sensitive line ratio & Assumed density $\left[\mathrm{cm}^{-3}\right]$ & Temperature $[\mathrm{K}]$ \\
\hline$[\mathrm{N} \mathrm{I}] 5197 \AA / 1.040 \mu \mathrm{m}$ & $10^{4.1}-10^{4.7}$ & $7000-16000$ \\
{$[\mathrm{~N} \mathrm{II}](6548+6583) / 5754 \AA$} & $10^{4.1}-10^{4.7}$ & $21000-52000$ \\
{$[\mathrm{O} \mathrm{I}](6300+6363) / 5577 \AA$} & $10^{3.7}-10^{4.7}$ & $<12000$ \\
{$[\mathrm{O}$ III $](4958+5006) / 4363 \AA$} & $10^{3.7}-10^{4.7}$ & $22500-25000$ \\
{$[\mathrm{~S} \mathrm{II}](6716+6730) / 4068 \AA$} & $10^{4.1}-10^{4.7}$ & $5000-12500$ \\
{$[\mathrm{~S} \mathrm{III}](0.906+0.953) \mu \mathrm{m} / 6312 \AA$} & $10^{3.7}-10^{4.7}$ & $39000-43500$ \\
\hline
\end{tabular}

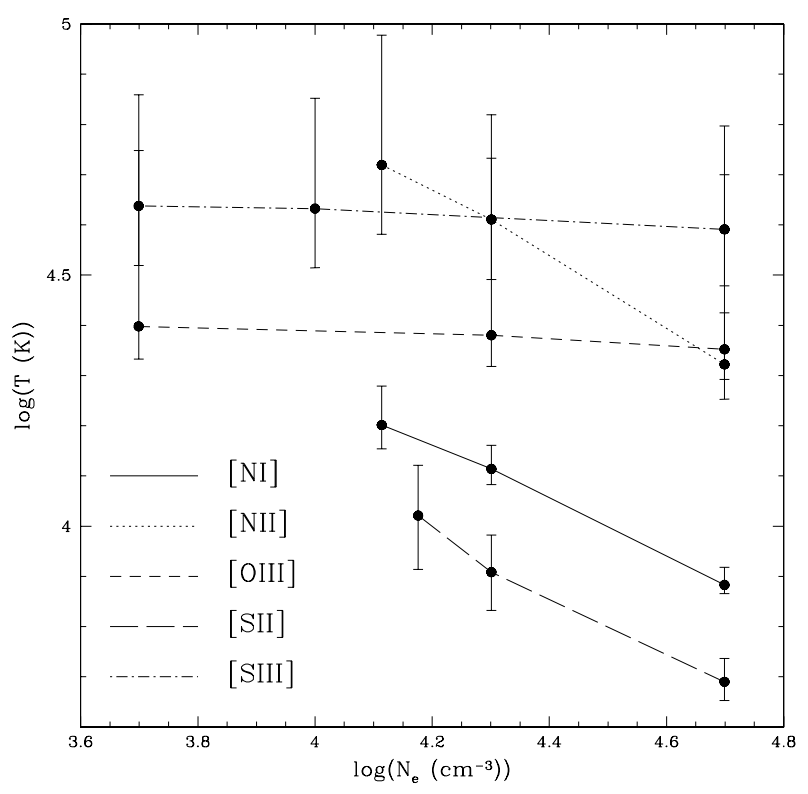

Fig. 5. Electron densities and temperatures found for Mrk 1210.

density $n_{\mathrm{e}}=50000 \mathrm{~cm}^{-3}$. Electron temperatures $T_{\mathrm{e}}>20000 \mathrm{~K}$ are difficult to obtain in the $\mathrm{O}^{+2}$ and $\mathrm{N}^{+}$zones in photoionized gas, requiring a source of energy input in addition to photoionization. Our working hypothesis is that this source is associated to shock heating.

Another well-known temperature diagnostic is the [S III] $(0.906+0.953) \mu \mathrm{m} / \lambda 6312$ line ratio, which is analogous to the $[\mathrm{O}$ III] $(\lambda 4958+\lambda 5006) / \lambda 4363$ ratio. Nonetheless, it is not commonly used, mainly due to the problem of deblending [S III] $\lambda 6312$ from the usually considerably stronger [O I] $\lambda 6300$. Our medium-resolution optical spectrum allowed us to separate these two lines without introducing large uncertainties in the line fluxes. From the [S III], we calculated the temperature of the $\mathrm{S}^{+2}$ region using the TEMDEN task of IRAF STSDAS nebular package. The results are also plotted in Fig. 5. Note that the calculation is relatively insensitive to density, indicating a gas temperature of about $40000 \mathrm{~K}$ for densities in the interval $10^{4}-5 \times 10^{4} \mathrm{~cm}^{-3}$. Osterbrock et al. (1990) determined the $\mathrm{S}^{+2}$ gas temperature for several Seyfert galaxies, finding temperatures from $10000 \mathrm{~K}$ to the extreme case of $26100 \mathrm{~K}$ for the high-ionization Seyfert 1 galaxy III Zw 77. The unusually high temperature found for the $\mathrm{S}^{+2}$ emitting region of Mrk 1210, together with the high temperature derived from the [O III] line ratio, shows further evidence that shocks contribute to the ionization structure in the NLR of this galaxy. Recall that these lines are the ones with a blueshifted broad component that we associated to the radio-knots observed in VLBA maps.

Also plotted in Fig. 5 are the electron temperatures in the $\mathrm{N}^{0}$ and $\mathrm{S}^{+}$zones, derived from the temperature sensitive [N I] $\lambda 5197 / 1.040 \mu \mathrm{m}$ and [S II] $(\lambda 6716+\lambda 6730) / \lambda 4068$ line ratios, respectively. The ratio $[\mathrm{OI}](\lambda 6300+\lambda 6363) / \lambda 5577$ also works as a temperature indicator. Although the line [O I] $\lambda 5577$ is definitely present in the optical spectrum of Mrk 1210, the profile is too noisy to measure it accurately. Therefore, we could only set an upper limit for the line flux, which we used to derive an upper limit of the [O I] emitting gas temperature of $12000 \mathrm{~K}$. The low temperature of the gas, in addition to the low ionization potential of the ion indicates that these lines are probably emitted in the same region as [S II] and [N I].

The results found from the different indicators (see Table 6) show that the NLR of Mrk 1210 cannot be characterized by a single value of temperature and density, but that presents a noticeable gradient in the physical conditions. The temperatures range from about $10000 \mathrm{~K}$ up to the unusual value of $40000 \mathrm{~K}$ determined from the [S III] lines, although the latter value may be misleading because of the contribution from shocks. The range of densities extends at least over three orders of magnitude, from a low density region $\left(n_{\mathrm{e}}=1000 \mathrm{~cm}^{-3}\right)$ in which the $[\mathrm{S} \mathrm{II}] \lambda 6716$, $\lambda 6730$ lines (and probably the $[\mathrm{NI}]$ and [O I] lines) are emitted to a high density region $\left(n_{\mathrm{e}}=10^{5.2} \mathrm{~cm}^{-3}\right)$ where the [Fe II] lines are emitted. Note that densities that high are plausible for the [Fe II] given that the critical densities of most if its transitions are in the interval $10^{4}-10^{5} \mathrm{~cm}^{-3}$ (Nisini et al. 2002).

\section{Summary and conclusions}

We have carried out a detailed study of the physical properties of the nuclear and extended emission line regions of the Seyfert 2 galaxy Mrk 1210. For this purpose, we presented spectroscopic observations simultaneously covering the interval $0.8-2.4 \mu \mathrm{m}$. The spectrum in the range $0.81-1.03 \mu \mathrm{m}$ is the first one published for this object. For that reason, all lines detected in this region were not previously identified in this source.

The spectra of Mrk 1210 display a plethora of emission lines from those emitted by molecular $\mathrm{H}_{2}$, prominent mostly in the $\mathrm{K}$-band, through low and medium ionization lines such as [CI], [S II], and [S III] to very high ionization lines of [S IX] and [Si X]. 
In addition, Mrk 1210 is characterized by a rich [Fe II] spectrum, not previously reported in the literature for other AGN. Extended emission of [S III], [Fe II], and $\mathrm{He}$ I is found up to a distance of $500 \mathrm{pc}$ from the nucleus.

The large amount of spectral information at medium resolution, covering the optical and NIR regions allows us to study the physical conditions in the inner few hundred parsecs of this galaxy. Our main results can be summarized as follows:

(i) From the depth of the ${ }^{12} \mathrm{CO}(6-3)$ overtone bandhead at $1.618 \mu \mathrm{m}$, we estimated that $83 \pm 8 \%$ of the $H$-band continuum has a stellar origin. This value improves on previous estimates, which claimed that at least $50 \%$ of the $H$-band continuum was attributed to the AGN and agrees with results found from optical spectroscopy. It also suggests the usefulness of the NIR in determining the percentage contribution of the stellar population to the integrated continuum emission. After correcting for the increased contribution of the stellar population to the continuum, Mrk 1210 continues to display one of the highest star-formation efficiency rates among water megamaser galaxies in spite of its relatively low molecular gas content. This result further supports previous findings by showing that the molecular lines in Mrk 1210 are mainly excited by UV heating from stars.

(ii) The analysis of the emission line profiles, both allowed and forbidden, shows that most prominent lines are characterized by a narrow $\left(F W H M \sim 500 \mathrm{~km} \mathrm{~s}^{-1}\right)$ component on top of a broad $\left(F W H M>1000 \mathrm{~km} \mathrm{~s}^{-1}\right)$ blue-shifted component $\left(\Delta V \sim 150 \mathrm{~km} \mathrm{~s}^{-1}\right)$. The latter seems to be associated to a nuclear outflow, instead of the hidden BLR supposedly present in previous NIR observations of this object. Moreover, the differences in form and width of the line profiles of [S III], [Fe II], and $\mathrm{H}_{2}$ imply that the emission seen from each line originates in different volumes of gas.

(iii) We examined the internal extinction affecting the NLR within the inner few hundred parsec using several indicators, including [S II] and [Fe II] line ratios. The results reveal a dusty AGN, while the extended regions are barely affected by dust, if at all. In the inner $250 \mathrm{pc}$, we found $E(B-V)=0.5$ from the $\mathrm{HI}$ lines ratios. From the [Fe II] line ratios, a larger extinction value, $E(B-V)=1.5$, was derived. This supports the hypothesis that the [Fe II] lines are formed in a separate region, different from the partially ionized zone that exist in AGN. At 250 pc from the center, no extinction is found from our data.

(iv) Electronic densities and temperatures for the nuclear gas of Mrk 1210 were determined by means of several diagnostic line ratios. The results found from the different indicators show that the NLR of Mrk 1210 cannot be characterized by a single temperature and density, since it presents a noticeable gradient in the physical conditions. The temperatures range from about $10000 \mathrm{~K}$ up to the unusual value of $40000 \mathrm{~K}$ determined from the [S III] lines. This very high temperature, together with the high temperature derived from the [O III] line ratio and the fact that these two lines display broad blueshifted components, are evidence that shocks contribute to the ionization structure of the NLR of this galaxy. The range of NLR gas densities extends over three orders of magnitude and points to a low density region $\left(n_{\mathrm{e}}=1000 \mathrm{~cm}^{-3}\right)$ in which the [S II] $\lambda 6716, \lambda 6730$ lines (and probably the [N I] and [O I] lines) are emitted, up to a high density region $\left(n_{\mathrm{e}}=10^{5.2} \mathrm{~cm}^{-3}\right)$ where the $[\mathrm{Fe} \mathrm{II}]$ lines are emitted.
Acknowledgements. This research was partly supported by the Brazilian agency CNPq (309054/03-6, 473465/2004-3) to ARA and the European Commission's ALFA-II program through its funding of the Latin-American European Network for Astrophysics and Cosmology, LENAC to X.M. The authors thank the referee Enrique Pérez for his useful comments that improve this manuscript. This research made use of the NASA/IPAC Extragalactic Database (NED), which is operated by the Jet Propulsion Laboratory, California Institute of Technology, under contract with the National Aeronautics and Space Administration.

\section{References}

Antonucci, R. 1993, ARA\&A, 31, 473

Bautista, M. A., \& Pradhan, A. K. 1998, ApJ, 492, 650

Braatz, J. A., \& Wilson, A. S. 1994, ApJ, 437, L99

Cardelli, J. A., Clayton, G. C., \& Mathis, J. S. 1989, ApJ, 345, 245

Cid Fernandes, R., Heckman, T., Schmitt, H., González Delgado, R. M., \& Storchi-Bergmann, T. 2001, ApJ, 558, 81

Cushing, M. C., Vacca, W. D., \& Rayner, J. T. 2004, PASP, 116, 362

Fraquelli, H. A., Storchi-Bergmann, T., \& Levenson, N. A. 2003, MNRAS, 341, 449

Greve, A., Castles, J., \& McKeith, C. D. 1994, A\&A, 284, 919

Guainazzi, M., Matt, G., Fiore, F., \& Perola, G. C. 2002, A\&A, 388, 787

Hamman, F. 1998, ApJ, 500, 798

Hamman, F., Sabra, B., Junkkarinen, V., Cohen, R., \& Shields, G. 2002, MPE Rep., 279, 121 [arXiv: astro-ph/0304564]

Heisler, C. A., \& De Roberties, M. M. 1999, ApJ, 118, 2038

Heisler, C. A., \& Vader, J. P. 1994, AJ, 107, 35

Heisler, C. A., \& Vader, J. P. 1995, AJ, 110, 87

Heisler, C. A., Lumsden, S. L., \& Bailey, J. A. 1997, Nature, 385, 700

Hes, R., Barthel, P. D., \& Hoekstra, H. 1995, A\&A, 303, 8

Ho, L. C., Filippenko, A. V., \& Sargent, W. L. W. 2003, ApJ, 583, 159

Keel, W. C., de Grijp, M. H. K., Miley, G. K., \& Zheng, W. 1994, A\&A, 283, 791

Keenan, F. P. 1991, Ap\&SS, 186, 277

Koski, A. T. 1978, ApJ, 223, 56

Lutz, D., Maiolino, R., Moorwood, A. F. M., et al. 2002, A\&A, 396, 439

Martini, P., Regan, M. W., Mulchaey, J. S., \& Pogge, R. W. 2003, ApJS, 146, 353

Middelberg, E., Roy, A. L., Nagar, N. M., et al. 2004, A\&A, 417, 925

Miller, J. S. 1968, ApJ, 154, L87

Nisini, B., Caratti o Garatti, A., Giannini, T., \& Lorenzetti, D. 2002, A\&A, 393, 1035

Nussbaumer, H., \& Storey, P. J. 1988, A\&A, 193, 327

Osterbrock, D. E. 1989, Astrophysics of Gaseous Nebulae and Active Galactic Nuclei (Mill Valey, CA: University Science Books)

Osterbrock, D. E., Shaw, R. A., \& Veilleux, S. 1990, ApJ, 352, 561

Pogge, R. W., \& Owen, J. M. 1993, OSU Internal Rep. 93-01

Pradhan, A. K., \& Zhang, H. L. 1993, ApJ, 409, L77

Raluy, F., Planesas, P., \& Colina, L. 1998, A\&A, 335, 113

Rayner, J. T., Toomey, D. W., Onaka, P. M., et al. 2003, PASP, 115, 362

Reunanen, J., Kotilainen, J. K., \& Prieto, M. A. 2002, MNRAS, 331, 154

Reunanen, J., Kotilainen, J. K., \& Prieto, M. A. 2003, MNRAS, 343, 192

Rieke, G. H., \& Lebofsky, M. J. 1985, ApJ, 288, 618

Rodríguez-Ardila, A., Pastoriza, M. G., Viegas, S., Sigut, T. A. A. \& Pradhan, A. K. 2004, A\&A, 425, 457

Schinnerer, E., Eckart, A., \& Tacconi, L. J. 1998, ApJ, 500, 147

Schlegel, D. J., Finkbeiner, D. P., \& Davis, M. 1998, ApJ, 500, 525

Schulz, H., \& Henkel, C. 2003, A\&A, 400, 41

Scoville, N. Z., Hall, D. N. B., Kleinmann, S. G., \& Ridgway, S. T. 1982, ApJ, 253, 136

Storchi-Bergmann, T., Cid Fernandes, R., \& Schmitt, H. R. 1998, ApJ, 501, 94 Storchi-Bergman, T., Winge, C., Ward, M. J., \& Wilson, A. S. 1999, MNRAS, 304, 35

Terlevich, R., Melnick, J., Masegosa, J., Moles, M., \& Copetti M. V. F. 1991, A\&AS, 91, 285

Thompson, R. I. 1995, ApJ, 445, 700

Tran, H. D. 1995, ApJ, 440, 578

Tran, H. D., Miller, J. S., \& Kay, L. E. 1992, ApJ, 397, 452

Turner, J., Kirby-Docken, K., \& Dalgarno, A. 1977, ApJS, 35, 281

Vacca, W. D., Cushing, M. C., \& Rayner, J. T. 2003, PASP, 115, 389

Veilleux, S., Goodrich, R. W., \& Hill, G. J. 1997, ApJ, 477, 631

Wampler, E. J. 1968, ApJ, 154, L53

Wampler, E. J. 1971, ApJ, 164, 1

Watanabe, M., Nagata, T., \& Sato, S. 2003, ApJ, 591, 714 
X. Mazzalay and A. Rodríguez-Ardila: Optical and NIR spectroscopy of Mrk 1210, Online Material p 1

\section{Online Material}


X. Mazzalay and A. Rodríguez-Ardila: Optical and NIR spectroscopy of Mrk 1210, Online Material p 2
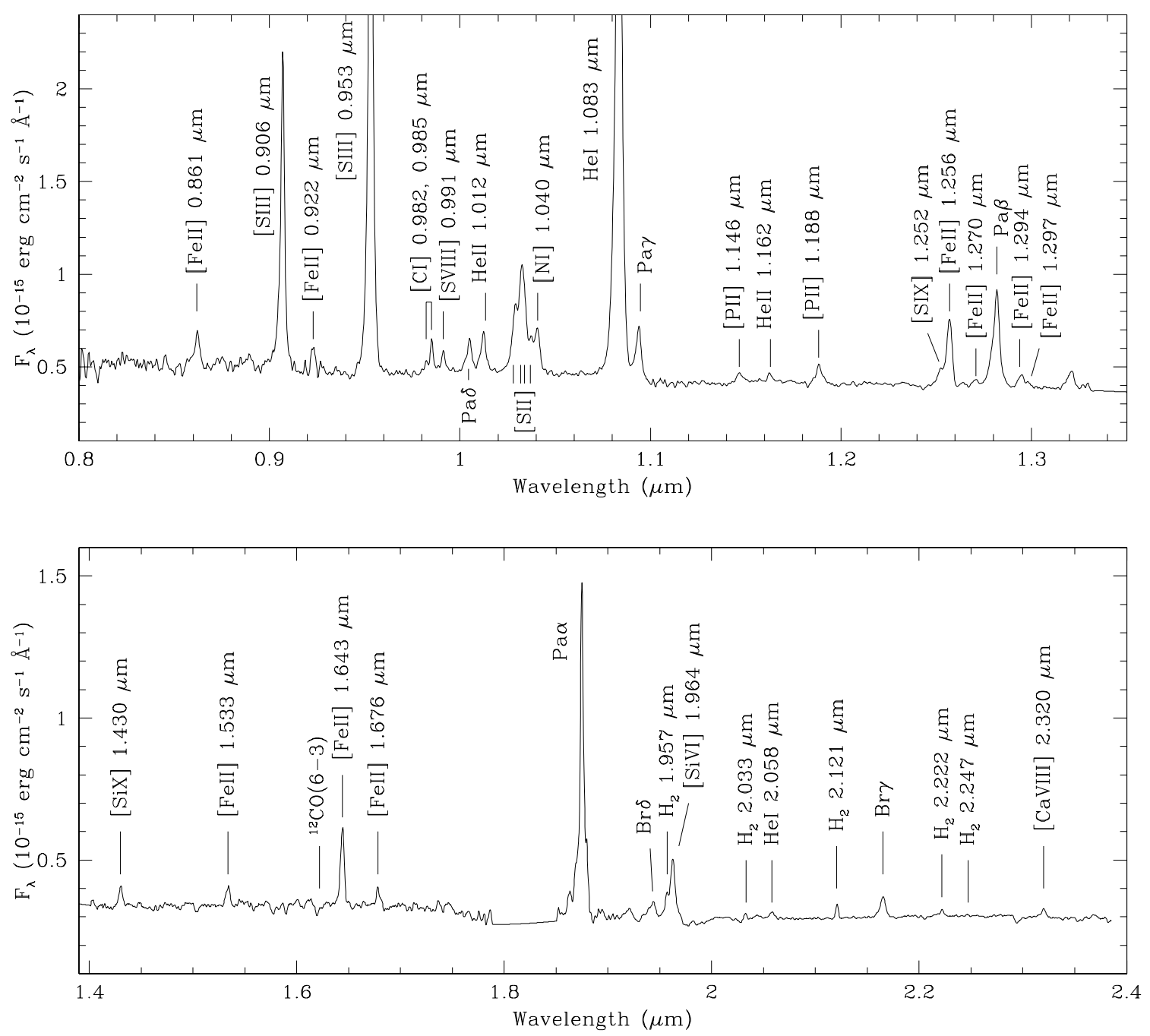

Fig. 1. Nuclear spectrum of Mrk 1210 in rest wavelength. The labels mark the position of the most important emission lines identified. 
X. Mazzalay and A. Rodríguez-Ardila: Optical and NIR spectroscopy of Mrk 1210, Online Material p 3

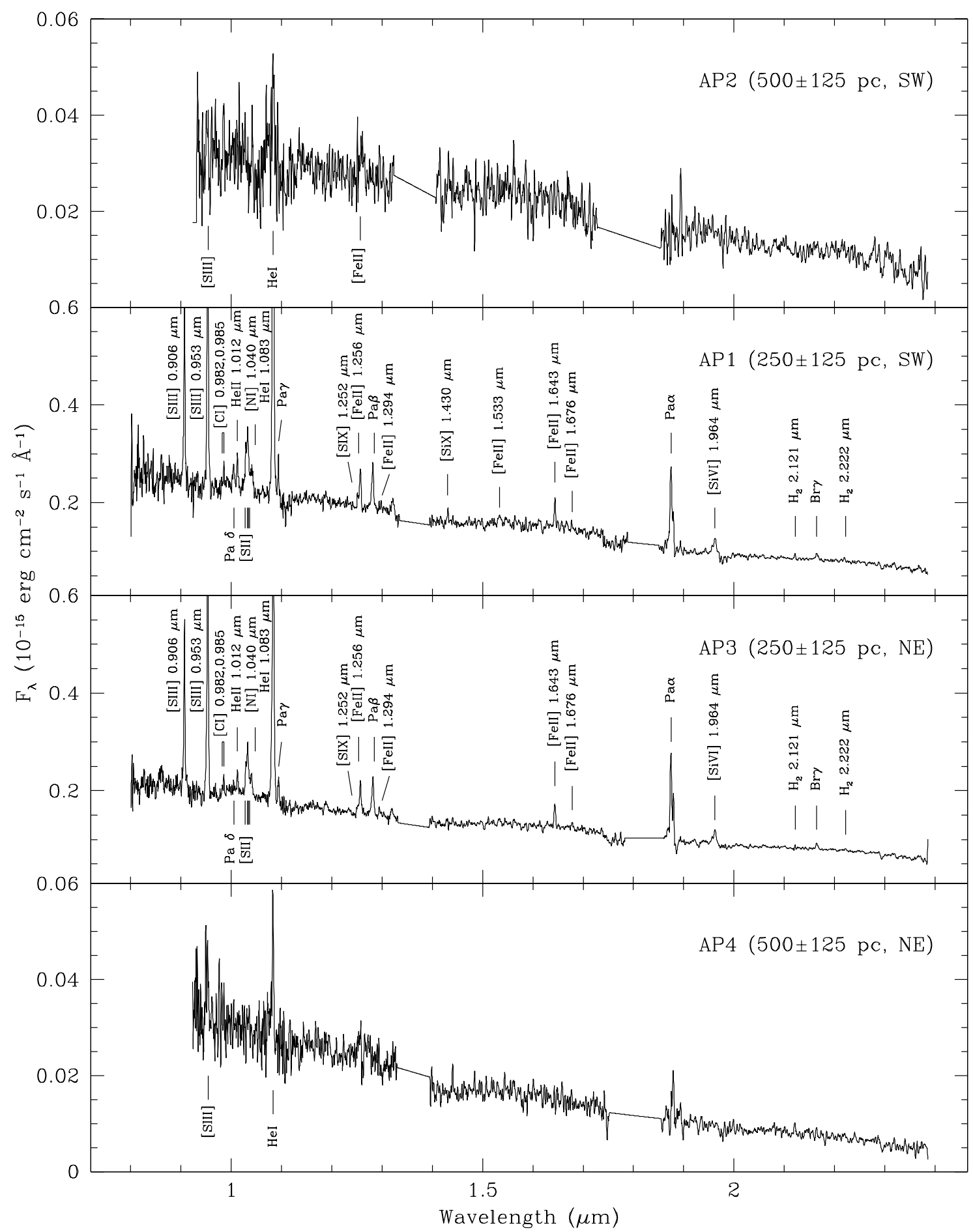

Fig. 2. Spectra of the extended emission region of Mrk 1210 in rest wavelength. Each panel represents the spectrum in a rectangular beam, which is $1^{\prime \prime}$ along the slit by the width of the slit $\left(0.8^{\prime \prime}\right)$. The position of the center of each spatial bin, relative to the nucleus, is indicated in the upper right of each panel. The second number is the aperture radius. 
X. Mazzalay and A. Rodríguez-Ardila: Optical and NIR spectroscopy of Mrk 1210, Online Material p 4
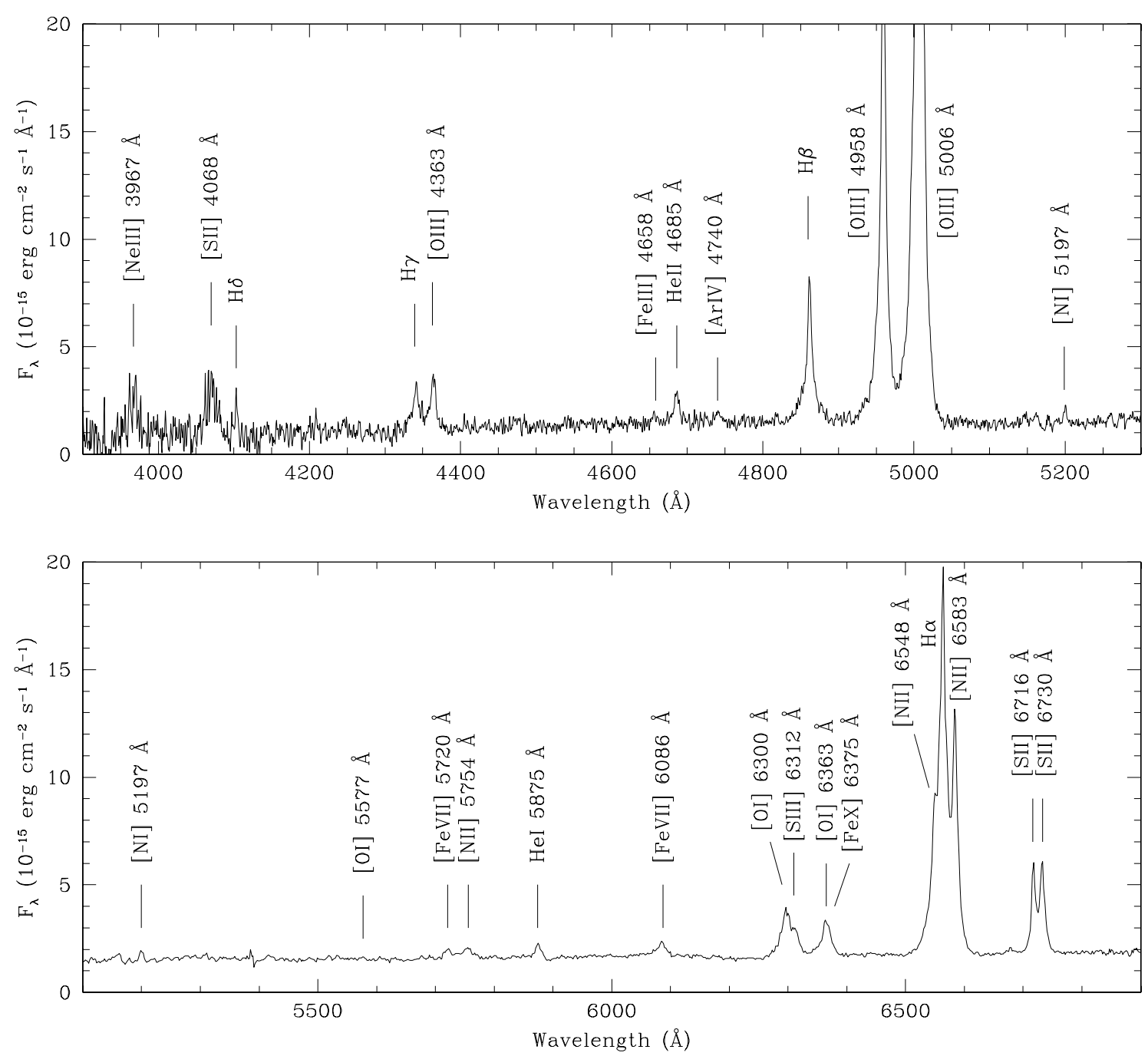

Fig. 3. Optical spectrum of Mrk 1210 in rest wavelength. The labels mark the position of the most important emission lines identified. 